\title{
Inserção do Psicólogo na Estratégia de Saúde da Família: Relato de Experiência na Residência Multiprofissional
}

\section{The Insertion of the Psychologist in the Family Health Strategy: An Experience Report on the Multidisciplinary Residency}

\author{
Inserción del Psicólogo en la Estrategia de Salud Familiar: Relato de Experiencia en \\ Residencia Multiprofesional
}

\author{
Ana Karoline Barros do Nascimento ${ }^{1}$ \\ Camila Aleixo de Campos Avarca \\ Escola de Saúde Pública do Ceará (ESP-CE)
}

\begin{abstract}
Resumo
O presente artigo tem por objetivo relatar a experiência de uma psicóloga em um Programa de Residência Multiprofissional a partir de sua inserção no Núcleo de Apoio a Saúde da Família (NASF), no município de Horizonte, CE. O objetivo é apresentar as ações desenvolvidas pela profissional residente em psicologia, de 2014 a 2016, e os desafios enfrentados na consolidação de práticas de base comunitária e territorial na Estratégia de Saúde da Família, a partir de três momentos: a) territorialização como estratégia estruturante de construção de agenda e ações em saúde com foco nas necessidades da população a partir de oficinas de planejamento participativo; b) execução das ações planejadas com foco na problematização dos processos de trabalho e c) potências e fragilidades desse processo. Como conclusão, apontamos a necessidade de se pensar novas formas de fazer da psicologia na Estratégia de Saúde da Família para além das suas práticas tradicionalmente esperadas.

Palavras-chave: Psicologia, saúde da família, residência multiprofissional, relato de experiência
\end{abstract}

\begin{abstract}
This article aims to report the experience of a psychologist in a Multidisciplinary Residency Program from its insertion at the Center of Support for Family Health (NASF) in the city of Horizonte, CE. The goal is to present the actions undertaken by the resident professional in psychology, 2014-2016, and the challenges faced in the consolidation of community and place-based practices in the Family Health Strategy, from three stages: a) territorial and structuring strategy schedule of construction and health actions focused on the needs of the population from participatory planning workshops; b) execution of the planned actions focusing on the critical work processes and c) powers and weaknesses of this process. In conclusion, we point out the need to think about new ways of doing psychology in the Family Health Strategy beyond its traditionally expected practices.

Keywords: Psychology, family health, multidisciplinary residency, experience report
\end{abstract}

\section{Resumen}

Este artículo tiene como objetivo presentar la experiencia de un psicólogo en un programa de residencia multidisciplinar desde su inserción en el Centro de Apoyo a la Salud de la Familia en la ciudad de Horizonte, estado de Ceará. El objetivo es dar a conocer las acciones del profesional residente en psicología, desde 2014 hasta 2016, y los desafíos encontrados en la consolidación de las prácticas comunitarias y territoriales de la Estrategia de Salud Familiar, a partir de tres etapas: a) territorialización como estrategia estructurante de construcción de agenda y acciones en salud centrados en las necesidades de la población de los grupos de planificación participativa; b) ejecución de las acciones prevista que se centran en problematizar los procesos de trabajo y c) poderes y debilidades de ese proceso. En conclusión, señalamos la necesidad de pensar en nuevas formas de hacer psicología en la Estrategia de Salud Familiar más allá de sus prácticas tradicionalmente esperadas.

Palabras clave: Psicología, salud de la familia, residencia multiprofesional, relato de experiencia

\footnotetext{
1 Endereço de Contato: Rua Major Cícero Franklin, 2027, Pacatuba, CE. CEP: 61800-000. E-mail: karolinebarros.n@hotmail.com
} 


\section{Introdução}

A Psicologia enquanto profissão no Brasil é recente: sua regulamentação no Brasil se deu em 1962, através da Lei n. 4.119 (Pereira \& Pereira, 2003). Inicialmente o foco principal da categoria era o trabalho clínico, individual, curativista, além de elitista, tal profissão não abarcava as esferas menores favorecidas (Carvalho \& Yamamoto, 2002).

A inserção da psicologia na saúde pública se inicia no final da década 1970, início da década de 1980. O contexto histórico estava marcado pelo golpe militar no país e por uma profunda crise econômica que afastava a classe média dos consultórios privados, permitindo, assim, a inserção da categoria em outros campos de trabalho (Dimenstein, 1998). Outro aspecto que favoreceu a inserção da psicologia nesse campo dizia respeito às críticas em relação à psicologia clínica tradicional por grupos acadêmicos que buscavam discutir a prática do psicólogo como agente transformador da sociedade: o intuito era motivar a novas práticas que tivessem uma relevância social (Carvalho \& Yamamoto, 2002).

O direito à saúde que, nesse momento, não era universal, a perspectiva hospitalocêntrica de cuidado e a desumanização nos atendimentos foram mote para o fortalecimento de movimentos sociais que se organizavam em favor da democratização do país, pelo direito à saúde e a outros direitos sociais, ficaram conhecidos como movimento de Reforma Sanitária e de Reforma Psiquiátrica (Paim, 2009). Todos esses movimentos sociais apontavam para uma necessidade de mudanças importantes no que diz respeito à saúde, pois era um momento de construção de uma nova consciência sanitária, de uma mudança do paradigma em saúde. Como consequência desses movimentos, o Sistema Único de Saúde (SUS) se materializa na Constituição Federal de 1988.

O intuito da criação do SUS era o de estabelecer um conceito ampliado de saúde, englobando as oportunidades de acesso aos serviços e ações voltados para prevenção, promoção e recuperação da saúde, legitimando assim o direito de todos a essas ações e compromisso do Estado na garantia dos mesmos.

Porém foi apenas depois da criação da Lei 8.080 e da Lei 8.142, ambas em 1990, que foi regulamentado o SUS, cujo modelo operacional foi então definido. Estabeleceram assim os princípios doutrinários do SUS: Universalidade, Equidade, Integralidade, Hierarquização, Descentralização e Participação Popular (Santos, 2013).

Com a implantação do SUS, surge a necessidade de formulação de políticas de saúde, de medidas governamentais voltadas para o fortalecimento da Atenção Básica de Saúde. Com esse intuito, o Ministério da Saúde lançou, em 2011, a Portaria MS/GM n. 2.488, que tinha como objetivo revisar as diretrizes e as normas para a organização da Atenção Básica para a ESF e o Programa de Agentes Comunitários de Saúde (Brasil, 2011). Com base nos princípios do SUS, surge o Programa de Saúde da Família, que posteriormente seria nomeado por Estratégia de Saúde da Família, com o intuito de substituir o modelo de atenção hegemônico e ampliá-lo para um programa de ações em saúde que visasse a um modelo de atenção mais integral (Costa \& Miranda, 2009).

Nesse contexto, a Estratégia Saúde da Família (ESF), aparece como um grande avanço do Sistema Único de Saúde (SUS), visando dar suporte assistencial a um número famílias de um determinado território, pelo qual a equipe irá assumir a responsabilidade sanitária. Essas 
equipes são compostas por profissionais de saúde, entre eles, médicos, enfermeiros, dentistas, técnicos de enfermagem e agentes comunitários de saúde (Brasil, 2006)².

A Estratégia de Saúde da Família (ESF) tem como objetivo o trabalho em equipe, a construção de vínculos com os usuários e a comunidade e o desenvolvimento de uma integração e mobilização intersetorial. Vale destacar que ela é a porta de entrada dos usuários no sistema de saúde (Camargo-Borges \& Cardoso, 2005).

Devido à complexidade e a maior necessidade de assistência à população, o Ministério da Saúde cria os Núcleos de Apoio à Saúde da Família (NASF), com o principal objetivo de apoiar a inserção da Estratégia Saúde da Família na rede de serviços, além de ampliar a abrangência e o escopo das ações da Atenção Básica e aumentar a resolubilidade desta, reforçando os processos de territorialização e regionalização em saúde (Brasil, 2009). As equipes dos NASF são constituídas por profissionais de diferentes áreas de conhecimento, que irão atuar de maneira integrada com as Equipes da Estratégia de Saúde da Família, trabalhando com o objetivo de integrar novas práticas de cuidado e saúde, de acordo com as necessidades de saúde do território, garantindo a continuidade e a integralidade da atenção (Brasil, 2009).

Vale destacar que mesmo o Núcleo de Apoio a Saúde da Família fazendo parte da Atenção Básica, estes não se constituem como serviços independentes das Equipes da Estratégia de Saúde da Família e tampouco como porta de entrada do sistema para os usuários (Brasil, 2011). Várias são as ações que podem ser desenvolvidas pelos profissionais do NASF, como por exemplo: construção de uma agenda de trabalho que dê prioridade às ações pedagógicas e assistenciais; educação permanente em saúde; ações intersetoriais; ações de prevenção e promoção de saúde, diretas e conjuntas com a ESF, dentre outras. Ressalta-se que essas ações tanto podem acontecer nas Unidades Básicas de Saúde, como em outros pontos do território (Brasil, 2011).

Tendo como referência o princípio da integralidade, que é a principal diretriz do SUS e que fomenta o trabalho em equipe do NASF, a psicologia aparece como uma das categorias que pode contribuir na ampliação do cuidado e na responsabilização compartilhada entre as equipes do NASF e as equipes da ESF. Essa inter-relação departamental possibilita uma atuação conjunta a fim de ampliar a visão sobre os sujeitos envolvidos, sobre suas famílias e comunidades, proporcionando um olhar para a saúde mental na Atenção Primária. Dessa forma, a Psicologia veio galgando essas novas demandas e tencionando novas formas de fazer.

Dentro desse contexto, o profissional de psicologia vem contribuindo com o seu olhar integrado, tanto no cuidado com a saúde mental, como na atenção básica, favorecendo uma compreensão do processo saúde-doença como algo multidimensional.

Dessa forma, pretendeu-se, com o presente artigo, relatar a experiência vivenciada por uma psicóloga em uma Residência Multiprofissional em Saúde da Família e Comunidade na cidade de Horizonte, CE. Este estudo se deu com o objetivo de se compreender a prática do psicólogo na Estratégia de Saúde da Família, a partir da experiência da Residência Integrada em Saúde da Escola de Saúde Pública do Ceará.

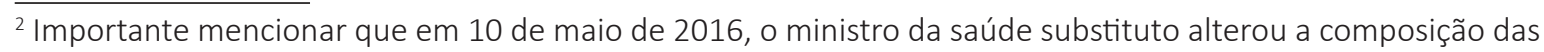
equipes de saúde da família: o gestor municipal poderá contratar o agente comunitário de saúde e/ou o técnico de enfermagem totalizando a soma de cargas horárias de 80 até 240 horas semanais. Cada equipe, portanto, poderá ter no mínimo dois ACS, e não mais quatro como eram recomendados.
} 


\section{Metodologia}

O trabalho em questão trata-se de um estudo descritivo, do tipo relato de experiência, construído a partir da vivência da autora enquanto residente de Psicologia no município de Horizonte, na Ênfase de Saúde da Família e Comunidade pela Escola de Saúde Pública do Ceará, dos anos de 2014 a 2016.

O município de Horizonte fica localizado na Região Metropolitana de Fortaleza, 40,1 km da capital cearense, foi emancipado do município de Pacajus há 26 anos e hoje é dividido em quatro distritos: Aningas, Dourado, Queimadas e Sede. Segundo o Instituto Brasileiro de Geografia e Estatística (IBGE), em 2015, Horizonte contava com aproximadamente 63.365 habitantes (IBGE, 2015).

No que diz respeito à Atenção Primária no município de Horizonte, a principal porta de entrada do Sistema Único de Saúde é composta por 19 Unidades Básicas de Saúde (UBS) com 20 Equipes de Saúde da Família e um NASF municipal (que não conta com profissional psicólogo). A turma de residentes da ênfase Saúde da Família e Comunidade que veio atuar como outra NASF no município é composta pelos seguintes núcleos profissionais: dois de enfermagem, três de fisioterapia, um de serviço social e um de psicologia. O cotidiano da construção da prática psicológica da residente no Núcleo de Apoio à Saúde da Família se deu em três Unidades Básicas de Saúde, atuando em duas áreas consideradas rurais e uma urbana, no centro da cidade.

Este relato de experiência tem por objetivo apresentar as ações desenvolvidas pela profissional residente em psicologia e os desafios que comumente apareceram nos cenários de prática, a partir de três momentos: a) territorialização como estratégia estruturante de construção de agenda e ações em saúde com foco nas necessidades da população; b) execução das ações planejadas com foco na problematização dos processos de trabalho e c) potências e fragilidades desse processo.

Em relação ao processo de territorialização, foram realizadas três oficinas de planejamento participativo em cada território ao longo de um mês, totalizando nove encontros ao todo.

Os encontros aconteciam em locais distintos: nas próprias UBS ou em espaços da comunidade, como em escolas, creches ou em centros culturais. O público-alvo da ação eram profissionais das UBS, trabalhadores da gestão em saúde e usuários do serviço/representantes da comunidade. Cada território contou com a presença de 15 a 30 pessoas desses segmentos.

As oficinas tiveram por objetivo conhecer e identificar o território em que iríamos trabaIhar e serviram de base para serem observadas as necessidades que emergiam na comunidade e incentivar no processo de autocuidado, tornando-os atores nos processos de saúde.

No primeiro momento, as oficinas foram pensadas para identificar quais eram essas as fortalezas, potencialidades, fraquezas e ameaças do território e qual o grau de urgência das questões trazidas pelo grupo. O segundo momento teve por objetivo identificar a governabilidade de cada eixo trazido, os problemas e as ameaças de cada proposição levantada e a quem competia à responsabilidade (gestão, equipe, comunidade ou dos três). No último encontro, foram discutidas propostas de como intervir nos problemas trazidos, a partir da reestruturação do processo de trabalho de todos envolvidos, cada qual com sua governabilidade e responsabilidade. 
A execução das ações planejadas e as potências e fragilidades desse processo serão descritos criticamente e reflexivamente a partir da sistematização das experiências vividas.

De acordo com Holliday (2006), a sistematização das experiências tem como objetivo reconstruir experiências, o que requer uma melhor compreensão do que foi vivido, para que possam ser retirados os ensinamentos e compartilhados com outras práticas similares a experiência vivenciada.

A sistematização das experiências vai produzir um novo conhecimento, com base nas práticas concretas, mas, para que possa ser produzido um novo conhecimento, é necessária uma análise crítica desta. Para sistematizar uma experiência é importante equilibrar dois caminhos o da descrição e o da teoria (Holliday, 2006).

\section{Descrição da Experiência}

Levando em consideração todo o caminho percorrido até aqui no que diz respeito à Psicologia e a Saúde Pública, na mudança do paradigma em saúde mais voltado para a integralidade das ações, na nova configuração de atenção a saúde, o Ministério da Saúde, junto com Ministério da Educação, desenvolveu as Residências Multiprofissionais em Saúde.

As Residências Multiprofissionais em Saúde foram regulamentadas pela promulgação da lei n. 11.129, de 30 de junho de 2005. Trata-se de um programa de pós-graduação em nível lato sensu, voltada para a educação em serviço, destinada às categorias profissionais que integram a área de saúde, com exceção da médica (Brasil, 2006). Trata-se de uma formação com duração de dois anos, carga horária semanal de 60 horas, que são divididas da seguinte forma: $80 \%$ que se concentram na prática assistencial e os $20 \%$ restantes englobam atividades teórico-práticas e teórico-conceituais. A carga horária total de 5.760 horas (Brasil, 2006).

Destacarei aqui a experiência vivenciada no município de Horizonte, a partir da segunda turma da Residência Integrada em Saúde, que iniciou suas atividades em maio de 2014 e contou com uma equipe de sete profissionais da Ênfase Saúde da Família e Comunidade sendo estas categorias: Enfermagem (2), Fisioterapia (3), Serviço Social (1) e Psicologia (1).

Inicialmente fomos divididos em duas equipes, uma enfermeira (ESF) e duas fisioterapeutas (NASF) que foram compor uma equipe já formada por residentes da turma 1 em Horizonte. Os outros profissionais, um fisioterapeuta, uma assistente social e psicóloga, iriam compor uma equipe de NASF, e a outra enfermeira que iria se inserir em outra equipe da ESF; é importante registrar que, nesses territórios, não existia a presença de residentes até então.

Dentro desse contexto e com base na Portaria n. 154, que apresenta duas modalidades de NASF, esse se caracterizaria como um NASF 2, que tem uma equipe mínima de três profissionais e se vincula com no mínimo três equipes de Saúde da Família (Brasil, 2009). Vale destacar que o município só contava com uma equipe de NASF municipal, responsável por dar assistência às demais Unidades Básicas de Saúde antes da chegada da Residência.

Por ora, as unidades de saúde que perfazem nosso cenário de prática consistiam de duas áreas consideradas rurais e uma urbana (Centro); definidos os cenários de atuação, iniciamos um processo de territorialização, que consiste em um instrumento importante e estratégico de organização dos processos de trabalho e das práticas de saúde, posto que as ações de saúde sejam implementadas sobre uma base territorial detentora de uma delimitação 
espacial. A territorialização já vem sendo utilizada no âmbito do SUS, mediante a incorporação de novos olhares e das questões de saúde ambiental e como se apresentam no território (Santos \& Rigotto, 2010). A territorialização se representa a partir da demarcação de áreas para o desenvolvimento das ações da Estratégia de Saúde da Família, bem como do reconhecimento das necessidades da população e os fatores que constituem barreiras de acessibilidade ao serviço. O processo de territorialização vai favorecer ainda a criação de vínculos entre os usuários e profissionais para que possam ter uma visão em sua totalidade do espaço onde atuam e trabalham para a promoção da saúde (Morais, Lacerda, Souza, Moreira, \& Souza, 2012).

Nesse sentido, a psicologia comunitária constitui um importante campo teórico-prático para o trabalho na atenção primaria à saúde, já que possibilita uma maior aproximação dos aspectos de relevância social das comunidades. A condição mais adequada para a psicologia comunitária é o trabalho educativo e de conscientização que possa visar à população promoção, prevenção e desenvolvimento socioeconômico (Starfield, 2002). Nesse sentido, amparada na postura defendida pela psicologia comunitária, pelos princípios do SUS, e ainda, como resultado do processo de territorialização, foram realizadas oficinas de planejamento participativo, sugerido pela equipe pedagógica da Residência, como parte do processo pedagógico desta.

Essas oficinas tinham o intuito de proporcionar conhecimento sobre o território, compreender as principais necessidades que a comunidade trazia, para que assim fosse possível se pensar uma agenda de trabalho que contemplasse essas necessidades. Para isso, foram convidados representantes da comunidade, profissionais das Unidades Básicas de Saúde e representantes da gestão: ao total, compareceram, ao longo do processo, cerca de 30 pessoas por oficina realizada.

As divulgações das oficinas foram feitas por meio de cartazes distribuídos nas Unidades Básicas de Saúde, convites feitos pelos Agentes Comunitários de Saúde e pela Equipe de Residentes na comunidade. Os locais escolhidos para a realização dessas oficinas foram em espaços da própria comunidade.

Para sistematizar, essas oficinas foram utilizadas como estratégia à matriz FOFA (Fortalezas, Oportunidades, Fraquezas e Ameaças), conhecida assim no Brasil, fundamentada por Kenneth Andrews e Roland Christensen como uma ferramenta estrutural da administração, com o intuito de identificar as principais ameaças, fraquezas, assim como as fortalezas e oportunidades (Tavares, 2008; Kotler, 1992).

Após a aplicação da matriz FOFA, foi utilizada outra ferramenta também conhecida na área da administração, conhecida como GUT (Gravidade, Urgência e Tendência), desenvolvida por Charles H. Kepner e Benjamim B. Tregoe em 1981, utilizadas para solução de problemas com o intuito de priorizar as ações com base na gravidade, na emergência e na tendência do fenômeno e assim planejar alternativas de ações. Essas ferramentas são utilizadas como base para a realização das oficinas de planejamento participativo (Sotille, 2014).

Como produto final dessas oficinas, alguns problemas tiveram maior destaque: a) aumento no consumo de drogas (trazendo como consequência um elevado índice de violência); b) ausência de atividades dirigidas tanto para os jovens como para os idosos e c) crescimento no número de adolescentes grávidas. Todos esses problemas apontados foram aspectos norteadores para a construção de uma agenda de trabalho. 
Outro aspecto importante na construção da agenda de trabalho foram algumas dimensões, sugeridas também como recurso pedagógico da instituição formadora, que davam conta de quatro dimensões, sendo elas a de prática clínica direta, prática clínica colaborativa, dimensão política e dimensão institucional. A primeira consistia em intervenções individuais e/ou coletivas, como visitas domiciliares, atendimentos individuais, salas de espera; a prática clínica colaborativa, que consistia em garantir turnos com ações em parceria com os profissionais da ESF ou com as demais categorias do NASF, como atendimentos e visitas domiciliares conjuntas, discussões de casos (Residência Integrada em Saúde, 2013).

Já a dimensão política baseava-se em ações com articulações intersetoriais, que estimulassem a participação popular, o controle social. A dimensão institucional diz respeito a um fortalecimento de uma gestão participativa. Vale destacar que essas dimensões foram criadas com o intuito de dar suporte e de facilitar o processo de construção da agenda (Residência Integrada em Saúde, 2013).

No que dizem respeito à psicologia, alguns turnos ficaram dedicados aos atendimentos individuais, entendo a importância do aporte clínico-individual na construção do cuidado em saúde mental, apesar do foco da psicologia no NASF não ser o atendimento clínico.

As visitas domiciliares e atendimentos conjuntos foram colocados com o intuito de identificar demandas já que muitos até aquele momento não entendiam muito bem o que a psicologia iria fazer além do atendimento individual e o que seriam demandas para o NASF. As visitas domiciliares foram potenciais tanto no que diz respeito à inserção na comunidade como no planejamento dos processos de intervenção. Para Góis (2008), é de suma importância não só o processo de inserção pela observação como também pela vivência do psicólogo no contexto desses usuários, moradores, de forma mais densa e comprometida. Para isso, ter um conhecimento acerca dos códigos, significados e sentido dados pelos moradores à sua realidade será de grande valia, para que seja possível uma compreensão de como eles lidam e vivenciam de acordo com seu cotidiano (Góis, 2008).

Os atendimentos conjuntos também foram muito importantes no que se refere a potencializar uma escuta qualificada, de desmistificar a figura do psicólogo como o único que pode realizar essa escuta, não, é claro, desvalorizando ou diminuindo toda a técnica construída durante a formação acadêmica, mas no sentido de que qualquer profissional poder realizar essa escuta, assim como também contribui com o aprendizado entre os profissionais sobre o saber um do outro. Segundo Mello Filho e Silveira (2005) uma ação de saúde interprofissional e interdisciplinar tem como objetivo integrar e promover uma troca de saberes entre os profissionais e assim permitir uma visão mais ampliada dos casos.

Cabe ressaltar que, inicialmente, tanto as consultas compartilhadas, como as visitas domiciliares tiveram o caráter de compreender as demandas territoriais, bem como estreitar o vínculo com a população do território, de modo a construir uma agenda de trabalho alinhada com a demanda da população. Em um segundo momento, entretanto, essas demandas partiam das reuniões de equipe e da solicitação dos profissionais da ESF, a partir da discussão de casos que demandassem a atuação do psicólogo.

No que diz respeito às dimensões institucional e política, foram criadas rodas de gestão que tinham o intuito de levantar os principais agravos, demandas tanto da comunidade, como questões relacionadas ao funcionamento das UBS, para discussão com a participação de representantes da gestão, da comunidade, profissionais das UBS que ocorriam men- 
salmente. Outras ações pensadas foram a participação em reuniões do conselho de saúde, articulações com equipamentos da Assistência Social, como o Centro de Referência da Assistência Social (CRAS) e Centro de Referência Especializado de Assistência Social (CREAS), com as escolas.

\section{Dificuldades e Reflexões}

Com a agenda construída e as intervenções no serviço já iniciadas, algumas dificuldades foram sendo percebidas logo no início: como já citado anteriormente, os profissionais e a população não enxergavam a psicologia para além do atendimento clínico.

Ressalta-se que, no município de Horizonte, não existem psicólogos na Saúde da Família que não fossem os residentes. Isso significa dizer que, até a entrada dos residentes no município, alguns profissionais da ESF não tinham tido a oportunidade de trabalhar com psicólogo junto à equipe. Isto é um fato que ainda repercute os inúmeros desafios colocados ao profissional de psicologia, no atual modelo de política de saúde (França \& Viana, 2006). Spink (2003) destaca essa dificuldade em seu estudo, discutindo a atuação dos profissionais que já atuavam na atenção a saúde e sua dificuldade ao compreender o papel dessas equipes multiprofissionais que vinham se constituindo e de como se daria esse processo na Atenção Básica. Nesse sentido, a autora discute a importância de o psicólogo definir novas formas de atuação, desconstruindo a discursividade de que a atuação clínica é seu papel central. Com o passar do tempo e como resposta aos atendimentos e visitas conjuntos, alguns profissionais foram percebendo que não só a psicologia, mas como as outras categorias poderiam contribuir muito com os cuidados aos pacientes.

Outra dificuldade encontrada está ligada a não adesão nos grupos pelos usuários. Reunimo-nos com as equipes das UBS com o intuito de buscar compreender a razão de não haver outros grupos ou de não existir alguns grupos-chaves no processo de educação em saúde com a população, como o grupo de gestantes. De acordo com os trabalhadores da UBS, os grupos não aconteciam porque não havia adesão da comunidade, por falta de interesse ou mesmo por comodidade. Muitos usavam o argumento do trabalho como o empecilho. Essa foi uma questão muito discutida pela equipe de residentes: como pensar estratégias que motivasse a comunidade a se envolver e de participar de forma mais ativa nesses processos e de inserir os profissionais da UBS nessas ações?

Nesse sentido, uma das estratégias em que tivemos êxito foi pensada com base em demandas trazidas na territorialização: os idosos reclamavam que não havia atividades de lazer, de exercício físico e que muitos ficavam ociosos. Em uma das áreas rurais, a que acompanhamos, foi possível observar muitos idosos com quadro de depressão, um número elevado que já tomava medicação há muito tempo, os quais só renovam receitas e não faziam sequer acompanhamento clínico.

Com base nisso, foi criado um grupo pelo fisioterapeuta que inicialmente tinha como foco o incentivo ao exercício físico. O público-alvo era os idosos hipertensos e diabéticos. Como estratégia de não deixar o foco apenas no corpo, mas de pensar nesse indivíduo de forma integral, a psicologia e o serviço social se inseriram nesse grupo, em encontros quinzenais para conversar sobre temas como estresse, depressão, direitos dos idosos e vários outros assuntos apontados por eles, com foco no desenvolvimento da autonomia destes. 
Ainda em relação às ações coletivas, foi possível desenvolver algumas parcerias com as Escolas e CRAS. Nesses espaços foram criados grupos de adolescentes com o intuito de dialogar sobre temas como sexualidade, gravidez na adolescência, drogas, projeto de vida, entre outros: novamente demandas encontras no processo de territorialização. Aqui vale destacar a relevância de promover a integração com a rede intersetorial a fim de fortalecer ações em saúde. Contudo essa é ainda uma fragilidade observada: a comunicação junto aos profissionais de outros serviços é algo que ainda necessita ser pensada com os profissionais da Estratégia de Saúde da Família.

As discussões de casos clínicos foram importantíssimas, a oportunidade de favorecer espaços de construção conjunta, que antes não existiam, foi primordial para possibilitar um diálogo entre as profissões, no qual todos estavam empenhados, cada um com seu saber, no cuidado com o usuário, na ampliação da clínica. O objetivo era ter uma atuação sustentada na clínica ampliada, onde fosse possível observar a responsabilidade sobre os usuários desses serviços de saúde, trabalhando com esse sujeito de forma singular, atuando de forma intersetorial, assumindo assim um compromisso ético (Brasil, 2004).

Em relação às demandas específicas de psicologia, as que inicialmente surgiram em maior escala foram as queixas sugestivas (tanto atendimentos individuais, quanto coletivos) de transtorno de ansiedade, principalmente em mulheres. A ideia, inicialmente, foi de criar um grupo de mulheres para que pudéssemos intervir tanto nessas questões como em outras que viessem aparecer, no sentido de acolher as demandas trazidas em relação à saúde mental. Entretanto não conseguimos agenciar a efetivação da proposta, por falta de pensar espaço e divulgação adequados para que as mulheres se interessassem em participar. Continuamos apenas nos atendimentos individuais em relação a essa demanda.

Andrade (2007) faz uma crítica ao foco nos atendimentos clínicos individuais prioritariamente e aponta para a necessidade de uma atuação voltada para atividades grupais, com ênfase nas demandas trazidas pela população. Destaca-se a importância das atividades de grupos no processo de fortalecimento de um coletivo, além da observação de que é possível, através de trocas de experiências, promovendo qualidade de vida à população.

Nesse sentido, outra fragilidade apontada no processo: durante o período da residência não foi possível a criação de grupos específicos de psicologia. Em contrapartida, houve a inserção em outros grupos específicos em andamento na UBS e no apoio as equipes da ESF, com orientações no sentido de acolhimento, as demandas em saúde mental, discussões de casos, foram potenciais no sentido de reconhecimento, valorização e contribuição da categoria nesses espaços.

De maneira geral, essa experiência teve um trabalho direcionado para ações de prevenção e promoção, no qual foi possível estabelecer uma parceria tanto com a comunidade, profissionais das Unidades Básicas de Saúde, como com os equipamentos e instituições do território e da rede em geral. Foi possível também observar que, dentre as ações de educação em saúde, as mais exitosas foram as ações com articulações intersetoriais, pois foram pensadas e planejadas a partir das demandas advindas do processo de territorialização.

Ainda é importante destacar que o fato de um dos autores ser um profissional residente, facilitou no que diz respeito a não ter como preocupação principal a produção, já que o foco era a formação e o aprendizado em serviço. Isso possibilitou abertura e liberdade para desenvolver as ações em geral. 


\section{Considerações Finais}

A inserção da Psicologia no contexto da Estratégia Saúde da Família (ESF) encontra alguns desafios por se tratar ainda de uma prática pouca conhecida por profissionais de saúde. Há o imaginário de que o trabalho do psicólogo é pautado apenas pelo atendimento clínico individual, reflexo da formação acadêmica da categoria insuficiente e pouco direcionado no que tange ao compromisso do (a) psicólogo (a) com o SUS e com a saúde da população.

A experiência vivenciada pela Residência demonstra que o papel do psicólogo e suas atribuições não estão devidamente formalizados; pelo contrário, vêm sendo delineadas e construídas com base nos impasses do cotidiano e nos enfrentamentos das dificuldades e dos problemas de saúde na Atenção Básica: ou seja, a partir do cotidiano da vida das pessoas. Dessa forma, amplia-se as práticas "psis", demonstrando o potencial de contribuição da categoria para uma visão integral do sujeito, demarcando assim um processo de consolidação neste campo de atuação, lembrando, é claro, de inúmeras possibilidades que ainda serão construídas com base nesse saber-fazer.

Vale destacar também a importante função da Residência Integrada em Saúde no sentido de contribuir para essa formação comprometida de profissionais para atuação no Sistema Único de Saúde.

\section{Referências}

Andrade, A. N. (2007). Práticas psicológicas, epistemicídio e unidades básicas de saúde. Psicologia Política, 13(1), 35-46.

Brasil. (2004). Ministério da Saúde. Secretaria Executiva. Núcleo Técnico da Política Nacional de Humanização. Humaniza SUS: A clínica ampliada. Brasília: Ministério da Saúde.

Brasil. (2006). Ministério da Saúde. Secretaria de Gestão do Trabalho e da Educação na Saúde. Departamento de Gestão da Educação na Saúde. Residência Multiprofissional em Saúde: Experiências, avanços e desafios. Brasília: Ministério da Saúde.

Brasil. (2009). Ministério da Saúde. Secretaria de Atenção à Saúde. Departamento de Atenção

Básica. Diretrizes do NASF: Núcleo de Apoio à Saúde da Família. Brasília: Ministério da Saúde.

Brasil. (2011). Secretaria de Atenção à Saúde. Departamento de Atenção Básica. Política Nacional de Atenção Básica. Brasília: Ministério da Saúde.

Camargo-Borges, C., Cardoso, C. L. (2005). A Psicologia e a Estratégia Saúde da Família: compondo saberes e fazeres. Revista Psicologia \& Sociedade, 17 (2), 26-32.

Carvalho, D. B., \& Yamamoto, O. H. (2002). Psicologia e políticas públicas de saúde: Anotações para uma análise da experiência brasileira. Psicologia para a América Latina, 1-8. Disponível em http://pepsic.bvsalud.org/scielo.php?pid= S1870-350X2002000100002\&script=sci_arttext\#not1

Costa, R. K. S. \& Miranda, F. A. N. (2009). Formação profissional no SUS: Oportunidades de mudanças na perspectiva da estratégia de Saúde da Família. Trabalho, Educação e Saúde, 6(3), 503-517.

Dimenstein, M. (1998). O psicólogo nas Unidades Básicas de Saúde: Desafios para a formação e atuação profissionais. Estudos de Psicologia, 3(1), 53-81. 
França, A. C. P., \& Viana, B. A.(2006). Interface Psicologia e Programa Saúde da Família- PSF: Reflexões teóricas. Revista Psicologia, Ciência e Profissão, 26(2), 246-257.

Góis, C. W. L. (2008). Saúde comunitária: Pensar e fazer. São Paulo: Hucitec.

Holliday, O. J. (2006). Sistematização das experiências: Algumas apreciações. Tradução de Sérgio Herbert. In: C. R. Brandão \& D. R. Streck (Orgs.), Pesquisa participante: O saber da partilha (pp. 227-243). Aparecida, SP: Ideias e Letras.

Instituto Brasileiro de Geografia e Estatística. (2015). Histórico dos Municípios: Horizonte.

Disponível em http://www.cidades.ibge.gov.br/painel/historico.php?lang=\&codmun= 230523

Kotler, P. (1992). Administração de marketing: Análise, planejamento, implementação e controle. São Paulo: Atlas.

Mello Filho, J., \& Silveira, L. M. (2005). Consulta conjunta: Uma estratégia de capacitação para a atenção integral à saúde. Revista Brasileira de Educação Médica, 29(2), 147-151. Disponível em http://www.educacaomedica.org.br/UserFiles/File/2005/volume29_2/ consulta_conjunta.pdf

Morais, A. J. D., Lacerda, M. K. S., Souza, S. C. O., Moreira, K. S., \& Souza, G. L. L. (2012). A territorialização na Estratégia Saúde da Família. In: Anais do 12 Congresso Brasileiro de Medicina de Família e Comunidade (p. 1431). Belém, PA.

Paim, J. S. (2009). Uma análise sobre o processo da Reforma Sanitária brasileira. Saúde em Debate, 33(81), 27-37.

Pereira, F. M., \& Pereira, N. A. (2003). O psicólogo no Brasil: Notas sobre seu processo de profissionalização. Psicologia em Estudo, 8(2), 19-27.

Residência integrada em saúde (2013). Texto base: Processo de trabalho e construção de agenda na ESF - Módulo pedagógico da RIS-ESP/CE. Disponível em http://cedes.esp.ce. gov.br/ead/file.php/156/Material_Didatico/Modulo_Transversal/Modulo_01/manual_ do_modulo_e_tutoriais/construcao_agenda/Texto-Base_Construcao_de_agenda_SF.pdf

Santos, A. L., \& Rigotto, R. (2010). Território e territorialização: Incorporando as relações produção, trabalho, ambiente e saúde na atenção básica à saúde. Trabalho, Educação e Saúde, 8(3), 387-406.

Santos, M. A. (2013). Lutas sociais pela saúde pública no Brasil frente aos desafios contemporâneos. Revista Katálysis16(2), 233-240.

Sotille, M. (2014). A ferramenta GUT- Gravidade, Urgência e Tendência. PM Tech Capacitação de Projetos. Disponível em http://www.portal-administracao.com/2014/01/analise-swot-conceito-e-aplicacao.html

Spink, M. J. P. (2013). Psicologia social e saúde: Práticas, saberes e sentidos. Petrópolis, RJ: Vozes.

Starfield, B. (2002). Atenção primária: Equilíbrio entre necessidades e saúde. Brasília: UNESCO, Ministério da Saúde.

Tavares, M. C. (2008). Gestão estratégica. São Paulo: Atlas. 
Recebido: 30/05/2016

Última revisão: 20/09/2016

Aceite final: 04/04/2017

\section{Sobre as autoras:}

Ana Karoline Barros do Nascimento: Psicóloga pela Universidade de Fortaleza (UNIFOR), especialista em Saúde da Família e Comunidade, na modalidade residência, pela Escola de Saúde Pública do Ceará (ESP-CE). E-mail: karolinebarros.n@hotmail.com

Camila Aleixo de Campos Avarca: Mestre em Psicologia Social pela Pontifícia Universidade Católica de São Paulo (PUC-SP), Especialista em Gestão de Serviços de Saúde pela Universidade Federal de São Paulo (UNIFESP), Coordenadora da ênfase de Saúde Mental da Residência Integrada em Saúde da Escola de Saúde Pública do Ceará (ESP-CE).E-mail: camilaavarca@hotmail.com 